\title{
Radial basis interpolation on homogeneous manifolds: Convergence rates
}

\author{
J. Levesley*and D. L. Ragozin ${ }^{\dagger}$
}

May 16, 2005

AMS(MOS) Classification: Primary 41A05; Secondary 41A15, 41A63

Keywords: Scattered data interpolation, homogeneous manifold, radial basis functions, zonal kernel, convergence rates

Running head: Radial interpolation on manifolds

\begin{abstract}
Pointwise error estimates for approximation on compact homogeneous manifolds using radial kernels are presented. For a $\mathcal{C}^{2 r}$ positive definite kernel $\kappa$ the pointwise error at $x$ for interpolation by translates of $\kappa$ goes to 0 like $\rho^{r}$, where $\rho$ is the density of the interpolating set on a fixed neighbourhood of $x$. Tangent space techniques are used to lift the problem from the manifold to Euclidean space, where methods for proving such error estimates are well established.
\end{abstract}

\section{Introduction}

There is currently significant interest in approximation on spheres, related to many interesting geophysical problems. There are a number of different approximation methods currently available on spheres, including wavelets [5], piecewise polynomial splines [1], and the subject of this paper, radial functions (sometimes called zonal splines or variational splines) [5, 8]. Error estimates and convergence rates for radial approximation on spheres, of an optimal nature, are recent in vintage $[6,7]$, and rely on some technically demanding mathematics. In this paper we build on an idea of Bos and de Marchi [3] in order to provide convergence rates for radial interpolation

\footnotetext{
* Supported by EPSRC Grant GR/L36222

${ }^{\dagger}$ Partially supported by NSF Grant DMS-9972004
} 
on a much wider class of manifolds: the reflexive, compact homogeneous spaces. In fact, much of what is accomplished here can also be achieved on a Riemannian manifold; see [9].

Let $M \subset \mathbb{R}^{d+k}$ be a $d$-dimensional embedded compact homogeneous $C^{\infty}$ manifold in the sense of Definition 1.1 in [11]. In particular there is a compact group $G$ of isometries of $\mathbb{R}^{d+k}$ such that for some $\eta \in M$ (often referred to as the pole) $M=\{g \eta: g \in G\}$. The reflexive condition means that for each pair $x, y \in M$ there is a $g \in G$ with $g x=y$ and $g y=x$. A kernel $\kappa: M \times M \rightarrow \mathbb{R}$ is termed zonal (or $G$-invariant) if $\kappa(x, y)=\kappa(g x, g y$ ) for all $g \in G$ and $x, y \in M$. Since the maps in $G$ are isometries of Euclidean space, they preserve both Euclidean distance and the (arc-length) metric $d(\cdot, \cdot)$ induced on the components of $M$ by the Euclidean metric. Thus the distance kernel $d(x, y)$ is zonal, as are all the radial functions, $\phi(d(x, y))$, which are kernels that depend only on the distance between $x, y$.

The manifold carries a unique normalised $G$-invariant measure which we call $\mu$ ( $\mu$ can be viewed either as the component of Lebesgue measure tangential to $M$ or as the measure induced by Haar measure on $G$ ). Then, we can define the inner product of real functions

$$
(f, g)=\int_{M} f g d \mu .
$$

An important part of analysing the interpolation process (and many other processes) is the construction of polynomials on the manifold. If $\Pi_{j}$ is the space of all polynomials of total degree $j$ in the ambient space $\mathbb{R}^{d+k}$ then $P_{j}:=\left.\Pi_{j}\right|_{M}$ is the space of degree $j$ polynomials on $M$. We can also construct sets of harmonic polynomials $H_{j}:=P_{j} \cap P_{j-1}^{\perp}$, where the orthogonality is with respect to the inner product $(\cdot, \cdot)$.

It is straightforward to show that $H_{j}$ is $G$-invariant, in the sense that $p_{j}\left(g^{-1} \cdot\right) \in H_{j}$ for all $p_{j} \in H_{j}$ and $g \in G$, and has an orthogonal decomposition into irreducible $G$-invariant subspaces (i.e., subspaces with no proper $G$-invariant subspace)

$$
H_{j}=\oplus_{l=1}^{h_{j}} \Xi_{j, l}
$$

Now, if $\left\{Y_{j, l}^{1}, \ldots, Y_{j, l}^{d_{j, l}}\right\}$ is an orthonormal basis for $\Xi_{j, l}$, then

$$
P_{j, l}(x, y)=\sum_{m=1}^{d_{j, l}} Y_{j, l}^{m}(x) Y_{j, l}^{m}(y), \quad x, y \in M,
$$


is the unique $G$-invariant reproducing kernel for $\Xi_{j, l}$. In other words, if $T_{j, l}$ is the orthogonal projector onto $\Xi_{j, l}$,

$$
\begin{aligned}
T_{j, l} f(x) & =\int_{M} P_{j, l}(x, y) f(y) d \mu(y), \quad x \in M, \\
\text { or } T_{j, l} f(x) & =\left(f, P_{j, l}(\cdot, x)\right), \quad x \in M,
\end{aligned}
$$

for all $f \in L_{2}(M)$. In particular,

$$
T_{j, l} f(x)=\left(T_{j, l} f, P_{j, l}(\cdot, x)\right), \quad x \in M .
$$

It is clear that $P_{j, l}$ is symmetric for all $j=0,1, \ldots$, and $l=1, \ldots, h_{j}$, i.e., $P_{j, l}(x, y)=P_{j, l}(y, x)$. The self-adjoint projectors $T_{j, l}$ associated with the $G$-invariant kernels $P_{j, l}$ are special cases of the general kernel operator $T_{\kappa}$ associated with a continuous zonal kernel $\kappa(x, y)$ by

$$
T_{\kappa} f(x)=\int_{M} \kappa(x, y) f(y) d \mu(y), \quad x \in M .
$$

All these operators are $G$-equivariant in the sense that

$$
\left[T_{\kappa} f\right]\left(g^{-1} x\right)=\left[T_{\kappa} f\left(g^{-1} \cdot\right)\right](x), \quad x \in M,
$$

and, at least on reflexive spaces, commute with each of the projectors $T_{j, l}$ (Proposition 3.7 in [11]). Since $\Xi_{j, l}$ is an irreducible $G$-invariant subspace, the commuting properties of the self-adjoint $T_{\kappa}$ imply $T_{\kappa} T_{j, l}=a_{j, l} T_{j, l}$ for some scalar $a_{j, l}$. Thus on reflexive spaces, by the density of polynomials, each zonal kernel $\kappa$ has an expansion (convergent as operators on $L_{2}(M)$ )

$$
\kappa(x, y)=\sum_{j=0}^{\infty} \sum_{m=1}^{h_{j}} a_{j, l} P_{j, l}(x, y) .
$$

In [11] the fundamentality of the set $\{\kappa(\cdot, y): y \in M\}$, of translates of a zonal kernel $\kappa$ in the space of continuous functions on $M$ is explored. The crucial result is that a zonal kernel has dense translates if and only if $a_{j, l} \neq 0$ for all $j, l$, i.e. the associated integral operator, $T_{\kappa}$ does not behave like the null operator on any of the $G$-invariant polynomial subspaces. As is shown in [9] the positive definiteness of a kernel $\kappa$ is equivalent to $\kappa$ being positive definite on each of these $G$-invariant polynomial subspaces. Here, $\kappa$ is positive definite if the quadratic form

$$
\sum_{x, y \in \Lambda}^{N} c_{x} c_{y} \kappa(x, y)
$$


is non-negative for any choice of finite data point set $\Lambda \subset M$ and coefficients $\left\{c_{x}: x \in \Lambda\right\}$. It is strictly positive definite if the above quadratic form is always positive for non-trivial coefficients and distinct data points.

A fixed strictly positive definite kernel $\kappa$ gives rise to a reproducing kernel Hilbert space $\mathcal{W}$, often called the native space. Given the values of $f$ on a set $\Lambda=\left\{x_{1}, \ldots, x_{N}\right\}$ of distinct points in $M$, we approximate $f \in \mathcal{W}$ by the $\kappa$-spline interpolant

$$
s_{\kappa}(x)=\sum_{y \in \Lambda} c_{y} \kappa(x, y)
$$

where the coefficients $\left\{c_{y}: y \in \Lambda\right\}$ are determined by satisfying the interpolation equations $s_{\kappa}(x)=f(x), x \in \Lambda$. This interpolation problem has a unique solution if $\kappa$ is strictly positive definite.

The reader should view the following work as tracking closely the corresponding analysis on the sphere, but we depart from the rationale of Light and v. Golitschek [6] as we try to convert error bounds to convergence rates. They describe a scaling operation on the sphere under which the Lagrange polynomials remain bounded, this being a key element of the analysis. The novelty of our approach is that we transplant the scaling, and in fact the entire error estimation onto the tangent space, and use well known properties of the scaling of Lagrange polynomials in Euclidean space. This is possible because distances on the manifold and its tangent space are comparable.

In Section 2 we develop the Hilbert space theory and show how to produce an error estimate for interpolation. In Section 3 we use this error estimate to produce convergence rates for positive definite kernels which are also radial (functions of geodesic distance on the manifold) or zonal. A number of the results in what follows are standard, but we include them for completeness.

\section{Hilbert Space, Reproducing Kernel, and Error Estimates}

In this section we describe the construction of a Hilbert space from a positive definite zonal kernel $\kappa$, and see that $\kappa$ is the reproducing kernel for this Hilbert space. This construction is very standard (see e.g. Atteia [2]), and is only included for completeness.

As indicated above, when $M$ is reflexive, every positive definite zonal kernel $\kappa$ whose translates are dense in $C(M)$ has an expansion in terms of 
the reproducing kernels for the minimal $G$-invariant polynomial subspaces of $L_{2}$ (see Theorems 12 and 16 in [9]):

$$
\kappa(x, y)=\sum_{j=0}^{\infty} \sum_{l=1}^{h_{j}} a_{j, l} P_{j, l}(x, y), \quad a_{j, l}>0 .
$$

For the rest of this paper we assume

$$
\sum_{j=0}^{\infty} \sum_{l=1}^{h_{j}} d_{j, l} a_{j, l}<\infty .
$$

Since $d_{j, l}=P_{j, l}(x, x)^{1 / 2} P_{j, l}(y, y)^{1 / 2} \geq\left|P_{j, l}(x, y)\right|$, this condition ensures that the positive definite zonal kernel defined by the sum above is jointly continuous, so that point evaluation makes sense.

Define the inner product

$$
[f, g]:=\sum_{j=0}^{\infty} \sum_{l=1}^{h_{j}} a_{j, l}^{-1}\left(T_{j, l} f, T_{j, l} g\right),
$$

where we recall that $(\cdot, \cdot)$ is the usual $L_{2}$ inner product. Let $\|f\|=[f, f]^{1 / 2}$. Then, the Hilbert space $\mathcal{W}$ is the algebraic subspace of $L_{2}$ satisfying

$$
\mathcal{W}:=\left\{f \in L_{2}:\|f\|<\infty\right\} .
$$

Condition (4) also ensures that $\kappa(\cdot, x) \in \mathcal{W}$ for every $x \in M$.

It is easy to show that point evaluation at $x \in M$ in this space is realised by inner product with $\kappa(\cdot, x)$ since

$$
\begin{aligned}
{[f, \kappa(\cdot, x)] } & =\sum_{j=0}^{\infty} \sum_{l=1}^{h_{j}} a_{j, l}^{-1}\left(T_{j, l} f, T_{j, l} \kappa(\cdot, x)\right) \\
& =\sum_{j=0}^{\infty} \sum_{l=1}^{h_{j}} a_{j, l}^{-1}\left(T_{j, l} f, a_{j, l} P_{j, l}(\cdot, x)\right) \\
& =\sum_{j=0}^{\infty} \sum_{l=1}^{h_{j}} T_{j, l} f(x) \\
& =f(x) .
\end{aligned}
$$

In the penultimate line we have used Equation (2).

The following result is a standard one so we state it without proof. 
Proposition 2.1 Let $s_{\kappa}$ be the $\kappa$-spline interpolant to $f \in \mathcal{W}$. Then $s_{\kappa}$ is the interpolant to $f$ from $\mathcal{W}$ with minimum norm.

Remark 2.2 In fact, one way of characterising the $\kappa$-spline interpolant is via a minimum norm property (see e.g. Light and Wayne [10] for details of such an approach).

The error estimate for $\kappa$-spline interpolation in $\mathcal{W}$ given next is also standard.

Theorem 2.3 Let $f \in \mathcal{W}$ and $s_{\kappa}$ be the $\kappa$-spline interpolant to $f$ on the finite point set $\Lambda$. Then, for $x \in M$,

$$
\left|f(x)-s_{\kappa}(x)\right| \leq\|f\| P(x, \Lambda),
$$

where

$$
\begin{aligned}
& P(x, \Lambda)= \\
& \min _{\left\{\nu_{y}: y \in \Lambda\right\} \subset \mathbb{R}}\left\{\kappa(x, x)-2 \sum_{y \in \Lambda} \nu_{y} \kappa(x, y)+\sum_{y, z \in \Lambda} \nu_{y} \nu_{z} \kappa(y, z)\right\}^{1 / 2} .
\end{aligned}
$$

\section{Pointwise Convergence Rates for Zonal Kernels}

In this section we consider zonal kernels and in particular radial kernels of the form

$$
\kappa(x, y)=\psi(d(x, y)),
$$

where $d(x, y)$ is the geodesic distance between $x$ and $y$, and $\psi: \mathbb{R} \rightarrow \mathbb{R}$ is a univariate, real-valued function. As we have already noted, the $G$-invariance of $d(x, y)$ shows radial kernels are clearly zonal. For the analysis in this section we will assume that $\kappa$ is positive definite and $2 r$-times continuously differentiable near the diagonal in $M \times M$.

We are interested in establishing (pointwise) convergence rates for the interpolants as the interpolation points $x_{1}, \ldots, x_{N}$ become dense about the point $x$. Since we will not be concerned with global constants, we are able to exploit the compactness of $M$ and simply give local estimates. By passing to the tangent space $T_{x}=\mathbb{R}^{d}$ at $x$ it becomes possible to reduce all the critical work to the Euclidean case.

Here are four simple results which lie at the heart of the Euclidean case. 
Lemma 3.1 Let $Z$ be $a \Pi_{2 r}$ unisolvent set in $\mathbb{R}^{d}$ with $\operatorname{card}(Z)=\operatorname{dim}\left(\Pi_{2 r}\right)$. Let $\left\{p_{z}: z \in Z\right\}$ be the Lagrange polynomials based on $Z$, i.e. $p_{z}(y)=\delta_{y, z}$. Then for any $\lambda>0$,

i. $\lambda Z:=\{\lambda z: z \in Z\}$ is unisolvent.

ii. The Lagrange polynomials based on $\lambda Z$ are $p_{\lambda z}=p_{z}(\cdot / \lambda), z \in Z$. In particular $p_{\lambda z}(0)=p_{z}(0)$.

Moreover, there exist $d_{0}>0$ and $C_{0}$ such that:

iii. Any set $\left\{\sigma_{z}: z \in Z\right\}$ with $\left\|z-\sigma_{z}\right\| \leq d_{0}$ is $\Pi_{2 r}$ unisolvent,

iv. If $S=\left\{\sigma_{z}: z \in Z\right\}$ is as in (iii) and $\left\{p_{\sigma_{z}}: z \in Z\right\}$ is a Lagrange basis for $\Pi_{2 r}$ based on $S$ then $\left|p_{\sigma_{z}}(0)\right| \leq C_{0}$.

Proof: The proof of (i) and (ii) are elementary once one has observed that the polynomials are invariant under scaling. As for (iii) and (iv), they follow directly from the continuity of the map from unisolvent sets of distinct centres to Lagrange polynomials.

The above lemma provides the basis for us to estimate the size of the analog of $P(x, \Lambda)$ for the Euclidean space case.

Proposition 3.2 Suppose $K$ is a strictly positive definite symmetric function on some compact neighbourhood $\bar{U} \times \bar{U}$ of $(0,0)$ in $\mathbb{R}^{d} \times \mathbb{R}^{d}$. Let $\mathcal{W}_{K \bar{U}}$ be the native space for $K$ on $\bar{U}$. Further suppose $K$ is in $C^{2 r}(\bar{U} \times \bar{U})$. Then there exist constants $\rho_{0}$ and $C>0$ such that for any finite set $V \subset U$ with

$$
\sup _{u \in \bar{U}} \min \|u-v\| \leq \rho<\rho_{0},
$$

the following estimate holds:

$$
\min _{\left\{\nu_{v}: v \in V\right\} \subset \mathbb{R}}\left\{\left\|K(\cdot, 0)-\sum_{v \in V} \nu_{v} K(\cdot, v)\right\|^{2}\right\} \leq C \rho^{2 r} .
$$

Proof: Fix a $\Pi_{2 r}$ unisolvent set $Z$ in $\mathbb{R}^{d}$ and let $d_{0}$ satisfy Lemma 3.1 (iii). Let $\rho_{0}$ be the largest number such that $\rho / d_{0} Z \subset U$ for all $\rho<\rho_{0}$. Such $\rho_{0}$ exists since $\bar{U}$ is a compact neighbourhood of 0 . Then, if $\rho<\rho_{0}$, using Lemma 3.1 (iii), for each $z \in Z$ we can choose distinct $v_{z} \in V$ such that $\left\|z-d_{0} v_{z} / \rho\right\| \leq d_{0}$ so that $V_{Z}=\left\{v_{z}: z \in Z\right\}$ is $\Pi_{2 r}$-unisolvent. Also, from Lemma 3.1 (iv) the associated Lagrange basis for $V_{Z},\left\{p_{v}: v \in V_{Z}\right\}$ satisfies $\left|p_{v}(0)\right| \leq C_{0}, v \in V_{Z}$. Moreover, since the set $Z$ is contained in ball of radius $C_{1} d_{0}$ say, $V_{Z}$ is contained in a ball of radius $C_{1} \rho$. 
The estimate (7) is obtained from Taylor's theorem as follows. The left hand side $(L H S)$ of $(7)$ is only increased by restricting the sums to be taken only over $V_{Z}$ instead of all of $V$. But then if $p_{v}, v \in V_{Z}$ are the Lagrange polynomials based on $V_{Z}$, setting $\nu_{v}=p_{v}(0)$ and using the reproducing properties of $K(\cdot, w)$ yields

$$
\begin{aligned}
\text { LHS } & \leq\left[K(\cdot, 0)-\sum_{v \in V_{Z}} p_{v}(0) K(\cdot, v), K(\cdot, 0)-\sum_{v \in V_{Z}} p_{v}(0) K(\cdot, v)\right] \\
& =\int_{\|\cdot\| \leq C_{1} \rho}\left(K(\cdot, 0)-\sum_{v \in V_{Z}} p_{v}(0) K(\cdot, v)\right) d\left(\delta_{0}-\sum_{v \in V_{Z}} p_{v}(0) \delta_{v}\right) .
\end{aligned}
$$

But the discrete measure in the last integral annihilates any polynomial $p \in \Pi_{2 r}$, since $p(0)=\sum_{v \in V_{Z}} p_{v}(0) p(v)$ by the Lagrange properties of $p_{v}$. In particular if $T_{2 r, w} \in \Pi_{2 r}$ is the Taylor polynomial for $K(\cdot, w)$ centred at $w$, then subtracting $T_{2 r, x}-\sum_{v \in V_{Z}} p_{v}(0) T_{2 r, v}$ from the last integrand changes nothing and shows

$$
\begin{aligned}
L H S \leq \int_{\|\cdot\| \leq C_{1} \rho}((K(\cdot, 0) & \left.\left.-T_{2 r, 0}(\cdot)\right)-\sum_{v \in V_{Z}} p_{v}(0)\left(K(\cdot, v)-T_{2 r, v}(\cdot)\right)\right) \\
& \times d\left(\delta_{0}-\sum_{v \in V_{Z}} p_{v}(0) \delta_{v}\right) .
\end{aligned}
$$

Since $K \in \mathcal{C}^{2 r}(\bar{U} \times \bar{U})$ and $\max _{v, w \in V_{Z} \cup\{0\}}\{\|v\|,\|v-w\|\} \leq 2 C_{1} \rho$ each of the remainder terms under the integral is $\mathcal{O}\left(\left(2 C_{1} \rho\right)\right)^{2 r}$ on the support of the discrete measure, where the order constant depends only on the $2 r$ 'th derivatives of $K(\cdot, w)$. Hence,

$$
\text { LHS } \leq(1+\Gamma) C_{2 r}\left(2 C_{1} \rho\right)^{2 r}(1+\Gamma),
$$

where $C_{2 r}$ is a constant depending only on the suprema of the $2 r$ 'th derivatives of $K$ which enter into the remainder in Taylor's formula, and $\Gamma=$ $\operatorname{card}(Z) C_{0}$.

To lift the analysis from the manifold to the tangent we need to show that the distances on each of these spaces are comparable.

Proposition 3.3 There is a compact set $U \ni 0$ such that for each $x \in M$ there is a one-to-one mapping $\phi_{x}: U \rightarrow M$, such that, for all $w, z \in U$,

$$
c_{1}\|w-z\| \leq d\left(\phi_{x}(w), \phi_{x}(z)\right) \leq c_{2}\|w-z\|,
$$

where constants $c_{1}, c_{2}$ are independent of $x$. 
Proof: Let us fix $m \in M$. Since $M$ is a manifold, there is an open neighbourhood $V \subset \mathbb{R}^{d}$ of the origin, and a one-to-one differentiable mapping $\phi: V \rightarrow \phi(V)$, where $\phi(V)$ is an open neighbourhood of $m$. Thus, there exists a compact convex set $U \subset V$, containing the origin, such that $\phi: U \rightarrow \phi(U)$ is a one-to-one differentiable mapping onto a compact neighbourhood of $m$.

We begin with the upper bound in this case. Let $w, z \in U$, and $\Gamma$ be the set of differentiable curves in $\mathbb{R}^{d}$ starting at $w$ and ending at $z$, parametrised on $[0,1]$. Then,

$$
\begin{aligned}
d(\phi(w), \phi(z)) & =\inf _{\gamma \in \Gamma} \int_{0}^{1}\left\|\frac{d}{d t} \phi(\gamma(t))\right\| d t \\
& \leq \int_{0}^{1}\left\|\frac{d}{d t} \phi((1-t) w+t z)\right\| d t .
\end{aligned}
$$

However, writing $\phi(z)=\left(\phi_{1}(z), \cdots, \phi_{d+k}(z)\right), z \in U$, we have $\frac{d}{d t} \phi((1-t) w+t z)=\left(\nabla \phi_{1}((1-t) w+t z) \cdot(w-z), \cdots, \nabla \phi_{d+k}((1-t) w+t z) \cdot(w-z)\right)$.

Thus, by Cauchy-Schwarz,

$$
\left\|\frac{d}{d t} \phi((1-t) w+t z)\right\| \leq\|w-z\|\|D \phi((1-t) w+t z)\|,
$$

where $D \phi(z)=\left(\left\|\nabla \phi_{1}(z)\right\|, \cdots, \nabla\left\|\phi_{d+k}(z)\right\|\right)$. Since $\phi$ is infinitely differentiable on $U$ we have

$$
d(\phi(w), \phi(z)) \leq c_{2}\|w-z\|,
$$

where $c_{2}$ depends only on $\phi$.

For the lower bound we start with the obvious bounds

$$
\begin{aligned}
d(\phi(w), \phi(z)) & \geq\|\phi(w)-\phi(z)\| \\
& \geq \max _{j=1, \ldots, d+k}\left|\phi_{j}(w)-\phi_{j}(z)\right| .
\end{aligned}
$$

Using the mean value theorem we have

$$
\left|\phi_{j}(w)-\phi_{j}(z)\right|=\|w-z\|\left|D_{w, z}\left(\nu_{l}\right)\right|,
$$

where $D_{w, z}$ is the directional derivative along the line segment connecting $w$ to $z$, and $\nu_{l}$ is some point along this line. Hence,

$$
d(\phi(w), \phi(z)) \geq\|w-z\| \max _{j=1, \ldots, d+k}\left|D_{w, z} \phi_{j}\left(\nu_{j}\right)\right| .
$$


Since $\phi$ is a chart the derivatives of the component functions cannot vanish simultaneously at a point. Thus, the last term on the right is a continuous function of two variables on the compact set $U \times U$, which is bounded away from zero. So, there exists a positive constant, depending only on $\phi$ such that

$$
d(\phi(w), \phi(z)) \geq c_{2}\|w-z\|
$$

for all $w, z \in C_{\sigma}$.

For arbitrary $x \in M$ there exists $g_{x} \in G$ such $g_{x} m=x$. Also, for any $g \in G$, and $y_{1}, y_{2} \in M, d\left(g y_{1}, g y_{2}\right)=d\left(y_{1}, y_{2}\right)$. We can now construct a chart $\phi_{x}: U \rightarrow N_{x}=\left\{g_{x} y: y \in \phi(U)\right\}$, a compact neighbourhood of $x$ :

$$
\phi_{x}(z)=g_{x} \phi(z) \text {. }
$$

Clearly,

$$
c_{1}\|w-z\| \leq d(\phi(w), \phi(z))=d\left(\phi_{x}(w), \phi_{x}(z)\right) \leq c_{2}\|w-z\|,
$$

and the constants $c_{1}, c_{2}$ are independent of $x$.

When we combine the purely local Proposition 3.2 with the metric equivalence result above the principal local error estimate for $s_{\kappa}$ spline interpolation is obtained.

Theorem 3.4 Let $\kappa$ be a strictly positive definite zonal kernel on $M \times M$. Assume $\kappa$ is $2 r$-times continuously differentiable. Let $x \in S \subset M$. Suppose that the interpolation point set $\Lambda \subset S$ satisfies

$$
\max _{y \in S} \min _{z \in \Lambda} d(y, z) \leq \rho,
$$

for some sufficiently small $\rho$. Then, for $f \in \mathcal{W}$,

$$
\left|f(x)-s_{\kappa}(x)\right| \leq C\|f\| \rho^{r},
$$

where $C$ is independent of $\rho$ and $f$.

Proof: Let $\pi_{x}$ be the projector onto the tangent space $T_{x}$ to $M$ at $x$, as in the preceding discussion. So we assume the $x \in T_{x}$ is the origin. Also if $\phi$ is a local inverse to $\pi_{x}$, then $K(w, z)=\kappa(\phi(w), \phi(z))$ defines a positive definite function on a compact neighbourhood of 0 , satisfying the hypotheses of Proposition 3.2. Now taking account of the metric stretching in Proposition 3.3, the result in (7) carries over to $M$. Combining this with Theorem 2.3 yields the result. 
Acknowledgements: We thank the referees for their careful reading of this paper and the subsequent suggestions they made which have improved our presentation.

\section{References}

[1] P. Alfeld, M. Neamtu, and L. L. Schumaker, Fitting scattered data on sphere-like surfaces using spherical splines, J. Comput. Appl. Math. 73 (1995), 5-43.

[2] M. Atteia, Hilbertian Kernels: Spline Functions, Studies in Computational Mathematics IV, North Holland, 1994.

[3] L. Bos and S. De Marchi, Limiting values under scaling of the Lebesgue function for polynomial interpolation on spheres, J. Approx. Theory 96 (1999), 366-377.

[4] N. Dyn, F. J. Narcowich, and J. D. Ward, Variational principles and Sobolev-type estimates for generalised interpolation on a Riemannian manifold, Constr. Approx. 15 (1999), 175-208.

[5] W. Freeden, On spherical spline approximation and interpolation, Math. Meth. in Appl. Sci. 3 (1981), 551-575.

[6] M. von Golitschek and W. A. Light, Interpolation by polynomials and radial basis functions on spheres, Constr. Approx. 17 (2001), 1-18.

[7] K. Jetter, J. Stöckler, and J. D. Ward, Error estimates for scattered data interpolation on spheres, Math. Comp. 68 (1999), 733-747.

[8] J. Levesley, W. A. Light, D. L. Ragozin, and X. Sun, A simple appraoch to the variational theory for interpolation on spheres, in New Developments in Approximation Theory, M. W. Müller, M. D. Buhmann, D. H. Mache and M. Felten (eds), ISNM 132, Birkhauser (1999), 117-143.

[9] J. Levesley and D. L. Ragozin, Positive definite kernel interpolation on manifolds: Convergence rates, Approximation Theory X, L. L. Schumaker and J. Stockler (eds), Vanderbilt Univ. Press (2002), 277-285.

[10] W. A. Light and H. Wayne, Error estimates for approximation by radial basis functions, in Wavelet Analysis and Approximation Theory, S.P. Singh and A. Carbone (eds), Kluwer Academic, Dordrecht (1995), 215246. 
[11] D. L. Ragozin and J. Levesley, The density of translates of zonal kernels on compact homogeneous spaces, J. Approx. Theory 103 (2000), 252268.

[12] R. Schaback, Multivariate interpolation and approximation by translates of a basis function, in Approximation Theory VIII, Vol 1, C.K. Chui and L.L. Schumaker (eds), World Scientific, Singapore (1995), 491-514.

J. Levesley

Department of Mathematics

University of Leicester

Leicester LE1 7RH, UK.

jl1@mcs.le.ac.uk

D. L. Ragozin

Department of Mathematics

Univ. of Washington

Box 354350, Seattle

WA 98195, USA.

rag@math . wash . edu 